\title{
QoE-Driven D2D Media Services Distribution Scheme in Cellular Networks
}

\author{
Mingkai Chen, ${ }^{1,2}$ Lei Wang, ${ }^{1,2}$ Jianxin Chen, ${ }^{1}$ and Xin Wei ${ }^{1}$ \\ ${ }^{1}$ Key Lab of Broadband Wireless Communication and Sensor Network Technology, \\ Nanjing University of Posts and Telecommunications, Ministry of Education, Nanjing, China \\ ${ }^{2}$ The State Key Laboratory of Integrated Services Networks, Xidian University, Xian, China \\ Correspondence should be addressed to Mingkai Chen; mingkaichen1989@163.com
}

Received 15 April 2017; Accepted 25 May 2017; Published 19 July 2017

Academic Editor: Feng Wang

Copyright (C) 2017 Mingkai Chen et al. This is an open access article distributed under the Creative Commons Attribution License, which permits unrestricted use, distribution, and reproduction in any medium, provided the original work is properly cited.

\begin{abstract}
Device-to-device (D2D) communication has been widely studied to improve network performance and considered as a potential technological component for the next generation communication. Considering the diverse users' demand, Quality of Experience (QoE) is recognized as a new degree of user's satisfaction for media service transmissions in the wireless communication. Furthermore, we aim at promoting user's Mean of Score (MOS) value to quantify and analyze user's QoE in the dynamic cellular networks. In this paper, we explore the heterogeneous media service distribution in D2D communications underlaying cellular networks to improve the total users' QoE. We propose a novel media service scheme based on different QoE models that jointly solve the massive media content dissemination issue for cellular networks. Moreover, we also investigate the so-called Media Service Adaptive Update Scheme (MSAUS) framework to maximize users' QoE satisfaction and we derive the popularity and priority function of different media service QoE expression. Then, we further design Media Service Resource Allocation (MSRA) algorithm to schedule limited cellular networks resource, which is based on the popularity function to optimize the total users' QoE satisfaction and avoid D2D interference. In addition, numerical simulation results indicate that the proposed scheme is more effective in cellular network content delivery, which makes it suitable for various media service propagation.
\end{abstract}

\section{Introduction}

With the propagation of the content delivery cooperative transmission technologies, D2D communication is considered as one of the most promising techniques for the next generation communication or new mobile applications, and it has recently received a substantial amount of interest [13]. Therefore, such D2D communication makes it possible to provide large heterogeneous media content services, such as HD (High Definition) video stream service, lossless music service, or website service for moving UE (User Equipment) [4]. Moreover, the heterogeneous media service over D2D communication is a very interesting topic. Meanwhile, it can make a difference on our daily life and provide a high Quality of Experience (QoE) service entertainment [5-7].

1.1. Related Work. At present, most of existing works on the content distribution for D2D communication underlaying cellular networks is focused on developing the transmission rate about the popularity files and $\mathrm{D} 2 \mathrm{D}$ transmission radius. In [8], by means of exploiting the optimal collaboration distance, Golrezaei et al. have proposed a novel scheme to increase the throughput of video files, which in the cellular networks proves the possibility of improving spectral efficiency. The new architecture to achieve wireless D2D cache collaboration is proposed in [9] from the viewpoint of asymptotic scaling characteristics and video content popularity. In [10], considering the improvement of the area spectral efficiency of video transmission, Shanmugam et al. present a small cells heterogeneous architectures. In addition, [11] studies and formulates a max-flow optimization problem to maximizes the content downloading flows in $\mathrm{D} 2 \mathrm{D}$ communication underlaying cellular networks and obtains the content downloading performance upper bound. Although this data dissemination protocols and schemes can be studied in D2D communication, which just focus 
on the maximum capacity, optimal relationship between $\mathrm{D} 2 \mathrm{D}$ radius, and service popularity for $\mathrm{D} 2 \mathrm{D}$ networks, they ignore the characteristics of different media service, mobile opportunistic environment, and D2D interference. Moreover, they deprive the cochannel interference of frequency reuse in D2D communications and the QoE is out of consideration in a dynamic network scheduling problem [12]. Essentially, D2D communication technology belongs to point-to-point communication technology, and there is a certain similarity with P2P (Peer-to-Peer) technology in the dynamic network topology and data processing. But D2D communication pays more attention to the scope of users local service, and the P2P service is based on IP network location. At the same time, D2D communication is for wireless physical approaching users, and P2P users are near for a wired or wireless network virtual location. Therefore, in the D2D communication we need to consider problems in wireless transmission issue, while the P2P service does not consider.

Based on our previous work [13], the problem of heterogeneous media service over D2D cellular networks includes the following items: (1) content QoE satisfaction: how do we distribute the media content service to adapt dynamic complex networks and achieve optimal user satisfaction? (2) cache update: how to update each UE's cache in the context of mobile opportunistic meeting environment without exceeding UE capacity or bandwidth resource? These two issues interact with each other; thus, the challenging problem concerning on QoE-driven network service has arisen across the dynamic networks and multiple UE [12]. Consequently, these issues may also perplex transmission quality of media service in the D2D networks. Therefore, we attempt to layout a suitable D2D distributed content distribution scheme actually corresponding to different QoE models.

1.2. Contributions. In this paper, we consider a media service content delivery issue through opportunistic D2D communication underlaying cellular networks and establish a diversified media services spontaneous propagation framework among cellular users. Our objective is to maximize the total user' QoE MOS value and achieve a balance between the amount of media services and cellular resource. We first identify an objective function that incorporates the different media characteristics and QoE model. Different from the traditional content distribution issue in the D2D networks where only the popularity of media service is considered, we here take additionally the channel impact of the different media service into account, in addition to the transmission packet loss rate of D2D direct link. Although there are some mature content delivery algorithms known in Peer-toPeer (P2P) networks, these algorithms are inadaptable to the media service in the mobile opportunistic environment and neglect the transmitting interference in the D2D network. The contributions of this paper are twofold as follows:

(1) We introduce the dynamic distributed heterogeneous Media Service Adaptive Update Scheme (MSAUS) for the D2D communication underlaying the cellular networks according to the popularity function and priority function. Then, we set up a D2D media service distribution scheme based on the popularity function and priority function to weigh the data dissemination characteristic and attempt to achieve the maximum satisfaction for cellular networks in the mobile opportunistic environment.

(2) We adopt resource greedy algorithm Media Service Resource Allocation (MSRA) to optimize the total users' satisfaction by reducing the interference from D2D media service update. This method reduces the impact of D2D interference caused by the band multiplexing on the prevalence media service.

1.3. Outline and Notation. The rest of this paper is organized as follows: Section 2 describes the media service system model for the cellular networks. In Section 3, we show an optimal distributed media service scheme based on the user cache and the popularity of media service. And the cellular resource allocation issue has been solved in Section 4. Then, extensive simulation results and comparisons are provided in Section 5. Section 6 provides conclusion and an outlook for future work. The following notations will be employed throughout the paper. Moreover, In Notations, we summarize the main notations used in this paper.

\section{System Model and Problem Formulation}

The scenario analyzed in this paper is depicted in Figure 1. There is a heterogeneous media service architecture for cellular and D2D communication networks, which contains three components, namely, D2D content delivery networks, network provider, and media content service provider [13]. In the D2D and cellular network component, a UE can access the BS (Base Station) via cellular link when the UE is under the base station coverage or chooses the vicinity UE to achieve the service through D2D link. In terms of the network provider part, the BS acts as gateway to connect the users to the media service providers. [8-10].

In our model, each user that is mobile and always connected to the $\mathrm{BS}$ is indicated as $u \in U \equiv\left\{u_{1}, \ldots, u_{i}\right\}$. Time is separated into time slots with a discrete index $t \in T$. During each time slot, we assume that the request media service is received with no errors and the media service updating can finish with no duration. Moreover, the BS controls the resource allocation in the D2D link and cellular link. According to the scared bandwidth resource $B \equiv\left\{B_{1}, \ldots, B_{i}\right\}$ the interferences should be one of the crucial factors to affect communication quality. Note that we assume the max number of interference source is $Z$. Then, the total bandwidth resource in the cellular network is expressed as

$$
B=\sum_{i=0}^{U} Z B_{i} .
$$

2.1. Media Service Request Generation Model. At the beginning of a time slot $t$, each user requests a random media service from a media service library $m \in M \equiv\left\{m_{1}, \ldots, m_{i}\right\}$. For each media service, we assume that it follows the Zipf distribution, which determines a ranking order of the media 


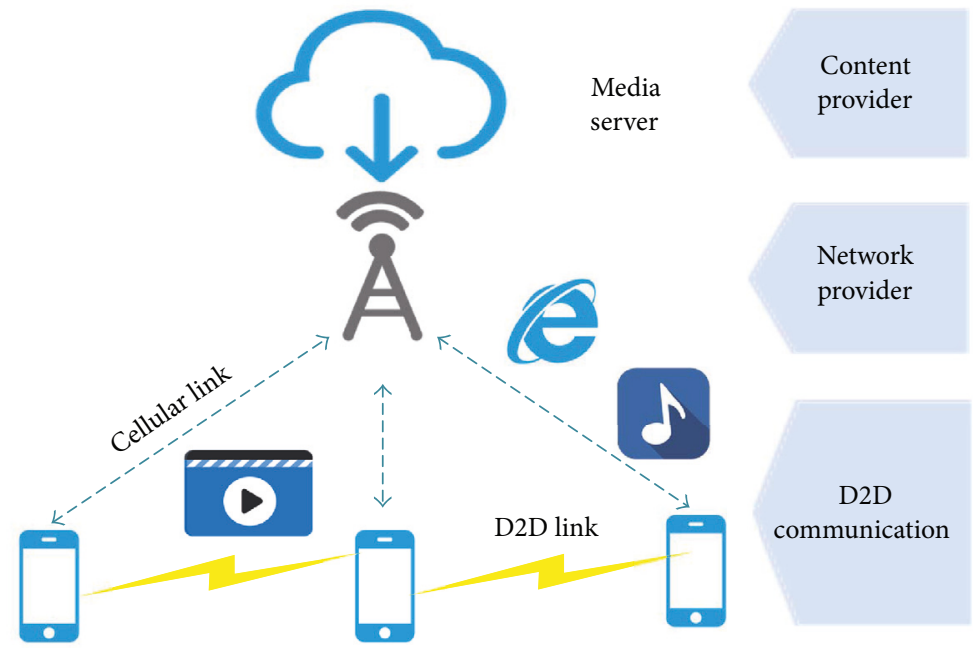

FIGURE 1: The architecture of media service for cellular networks.

TABLE 1: Different function expressions under different model of QoE functions.

\begin{tabular}{lccc}
\hline Model & T1: Best Effort Service & T2: Video Service & T3: Audio Service \\
\hline QoE function & $0.775 \log (R)+1.268$ & $\frac{2.797+30 \times 0.0065+0.2498 \ln (R)}{1+2.273 \Theta+7.1773(\Theta)^{2}}$ & $\frac{2.2073 \log (R)}{7.1773(\Theta)^{2}}$ \\
Priority function $\varphi$ & $\frac{\exp \left(-\lambda \pi r^{2} X_{i}\right)}{Q(R(d))}$ & $\frac{\exp \left(-\lambda \pi r^{2} X_{i}\right)}{Q(R(d), \Theta(d))}$ & $\frac{\exp \left(-\lambda \pi r^{2} X_{i}\right)}{Q(R(d), \Theta(d))}$ \\
Popularity function $\phi$ & $\frac{c|U| \exp \left(-\lambda \mathrm{SRC}_{m, i}\right)}{\operatorname{SRC}_{m, i} Q(R(d))}$ & $\frac{c|U| \exp \left(-\lambda \mathrm{SRC}_{m, i}\right)}{\mathrm{SRC}_{m, i} Q(R(d), \Theta(d))}$ & $\frac{c|U| \exp \left(-\lambda \mathrm{SRC} \mathrm{C}_{m, i}\right)}{\mathrm{SRC}_{m, i} Q(R(d), \Theta(d))}$ \\
\hline
\end{tabular}

service popularity. This assumption has been widely used [8] to describe content popularity distribution. According to the Zipf distribution, the popularity that a service $m_{i}$ is requested by a user $u_{i}$ is given by

$$
m_{i}=\frac{1 / i^{\gamma}}{\sum_{j=1}^{M}\left(1 / j^{\gamma}\right)}, \quad 1 \leq i \leq M, 1 \leq j \leq M,
$$

where $\gamma$ is a fixed parameter that describes the skewness of media service popularity, which defines the correlation level of user requests. High values of $\gamma$ mean that most of the requests are generated from a few most popular files. For a user making a random request, $m_{i}$ can be seen as the probability that the requested file is in the media service library $M$. If $\gamma=0$, all media services have the same request probability, while, in the case of high values of value of $\gamma$, most of the service are requested with a low probability and there are only a few popular files.

2.2. QoE Description Model. In the cellular networks, we consider the user QoE gain generated by a media service request $m$ from user $u_{i}$. Each user $i$ demands media services in many various requests. Meanwhile, the different media service types are considered as an important metric to efficiently allocate resource to heterogeneous multimedia traffic. The QoE function reflects the relative satisfaction level of a user regarding the allocated resources. Due to the process of demands for different services and QoE model functions, the heterogeneous media service reflects the diversified QoE models. Since users may demand heterogeneous media services, the flexible models are required for us to account for their MOS. In this paper, we define QoE functions to characterize the users' experience for different types of media service delivery. We consider the following usage media service type for D2D applications: (T1) Best Effort Service (BES): non-real-time service, such as file download or data transmission; (T2) Video Model: HDTV signal transmission, video on demand; (T3) Audio Model: digital radio broadcasting, lossless music service $[6,7,14]$. We take advantage of the different QoE function in the previous work to quantify the user's satisfaction [15], which are shown in the Table 1.

According to previous studies [15], the media service over cellular networks is greatly impacted by the transmission rate $R$ and PER (Packet Error Rate). Since the media service always prefers fulfilling user's demand as soon as possible, we assume that the D2D link rate $R_{D}$ is higher than the cellular link rate $R_{c}$ in the cell to achieve the higher user's satisfaction.

Then, the maximal achievable average data transmission rate for the downlink between the BS and UE $u_{i}$ denoted by $R_{c}$, as follows:

$$
R_{c}=B_{i} \log _{2}\left(1+\frac{p_{c} d_{i}^{-\alpha} h_{c}{ }^{2}}{N_{0}}\right),
$$

where $p_{c}$ means the transmission power from BS. Let $B_{i}$ denote the set of all D2D communication pairs in the cell and 
allocated bandwidth resource. Since D2D communication pairs share the same spectrum of the cellular uplink, we should consider the interference between all the different $\mathrm{D} 2 \mathrm{D}$ pairs and the average transmission rate of the $\mathrm{D} 2 \mathrm{D}$ pairs, denoted by $R_{D}$.

$$
R_{D}=B_{i} \log _{2}\left(1+\frac{p_{d} d^{-\alpha}{ }_{u_{i}, u_{j}}\left|h_{d}\right|^{2}}{\sum_{z \in Z} p_{d} d^{-\alpha}{ }_{u_{i}, u_{k}}\left|h_{d}\right|^{2}+N_{0}}\right),
$$

where $p_{d}$ is defined as the transmission power from UE. At this time, we assume that neither the transmitter nor the receiver discards packets maliciously; $u_{i}$ has received the signed ACK packet to ensure that $u_{i}$ successfully received the media service from the user $u_{j}$. A successful transmission probability from $u_{i}$ and $u_{j}$ is related to the transmission bit $R_{d}$ or $R_{c}$, packet exponent $\Lambda$, and the max number of interference source $Z$ [16]. Then, the probability of PER is given by

$$
\Theta(z, R)=1-\left(1-f\left(\frac{\Lambda}{R}\right)\right)^{z} \cdot f\left(\frac{\Lambda}{R}\right)^{(Z-1-z)}
$$

where

$$
f\left(\frac{\Lambda}{R}\right)=e^{-\Lambda / R}\left(\frac{\Lambda}{R}\right)
$$

2.3. Mobility Model. The user mobility pattern is modeled as a Point Poisson Process (PPP) model. A UE itself cannot know the probability of the UE it meets without the help of a BS control. Specifically, at the beginning of each time slot, each UE picks up a walking direction $\theta \in[0,2)$ randomly and independently. The UE also chooses a constant velocity $v$ to move during the rest of the time slot. They can be corresponded with other users during the mobility process. In this work, we assume the same mobility pattern for all users. Therefore, in each new time slot, the total users moving directions will refresh independently. Thus, the device random mobility is a Poisson distribution in the cellular network with density $\lambda$ everywhere; therein $\lambda$ is related to the number of users $U$ in the cellular networks [15].

Therefore, under this three condition, users' QoE value expressions are not the same under this heterogeneous and mobile media service situation. To depict the real cellular network environment, we define $X_{m, i}=1$ if UE $u_{i}$ is in possession of service $m$, and otherwise. In addition, we assume that all UE has the same cache size $c$. The matrix $X_{i \in r}=\sum_{r}\left(X_{m, i}\right)$ represents the state of the distributed cache under $\mathrm{UE} i$ 's D2D radius $r$ coverage.

Generally, we define $Q_{m, i}(\chi)$ to be the expected QoE gain generated by a request for service $m$ from user $i$. Hence, we denote $Q(\chi)$ as MOS function for the media service, which represents the score of QoE. The problem of D2D resource allocation for QoE-driven media service distribution can be explicitly formulated as

$$
\begin{array}{ll}
\max & Q(\chi)=\sum_{m \in M} \sum_{i \in U} P_{d} Q_{m, i}(\chi) X_{i \in r}+P_{c} Q_{m, i}(\chi) \\
\text { s.t. } & \sum_{m \in M} X_{m, i} \leq c \\
& B=\sum_{i=0}^{U} Z B_{i} \\
& X_{m, i} \in\{0,1\} \quad \forall i \in U \forall m \in M .
\end{array}
$$

Here, $P_{d}$ and $P_{c}$ are presented as the request distribution of D2D communication or cellular link, separately. Thus it can be seen that the total users QoE gain is affected by the user cache allocation, $X_{m, i}$, the media service popularity, $P_{c}$ and $P_{d}$, and the bandwidth resource, $Z$ and $B_{i}$. As a consequence, this media service problem is coupled with media content placement, user cache update and resource allocation. In the rest of the paper, we propose a distributed D2D media service scheme where each user and media service combine together to solve this issue through efficient cooperation.

\section{Distributed Media Service Scheme in D2D Communication}

In this section, we propose a mobile D2D data dissemination based on different QoE popularity function and priority function. In particular, we assume the UE may encounter with each other in an opportunistic way. Suppose that meetings among UE follow independent and memoryless processes. This helps us to find the optimal QoE scheme before evaluating them for these complex networks.

Due to the variable cellular network, the user's connection is based on the D2D communication radius. Meanwhile, the UE cannot clearly comprehend the status of transmit channel. The UE should attempt to achieve the most valuable media service against to the scarce resource and the limited cache storage. Therefore, when UE seeks the appropriate media service to update in the cellular network, we assume UE does not consider the interference in order to improve user satisfaction greatly. According to QoE analysis mentioned above, the media service's priority function is based on $R$ and $\Theta$, which is related to the Euclidean distance $d$. For PPP distribution in the cellular network with density $\lambda$ [17], the probability that in a slot there are $u$ devices in the D2D communication area is

$$
P(u, r, \lambda)=\frac{\left(\lambda \pi r^{2}\right)^{u}}{u !} e^{-\lambda \pi r^{2}} .
$$

Therefore, for a UE in the network the probability of at least another user caching to obtain the requested media service $m_{i}$ within the $\mathrm{D} 2 \mathrm{D}$ communication range and $c$ size cache is

$$
P_{m}^{\mathrm{D} 2 \mathrm{D}}=1-P(u, r, \lambda)=1-e^{-\lambda \pi r^{2} m_{i} c} .
$$




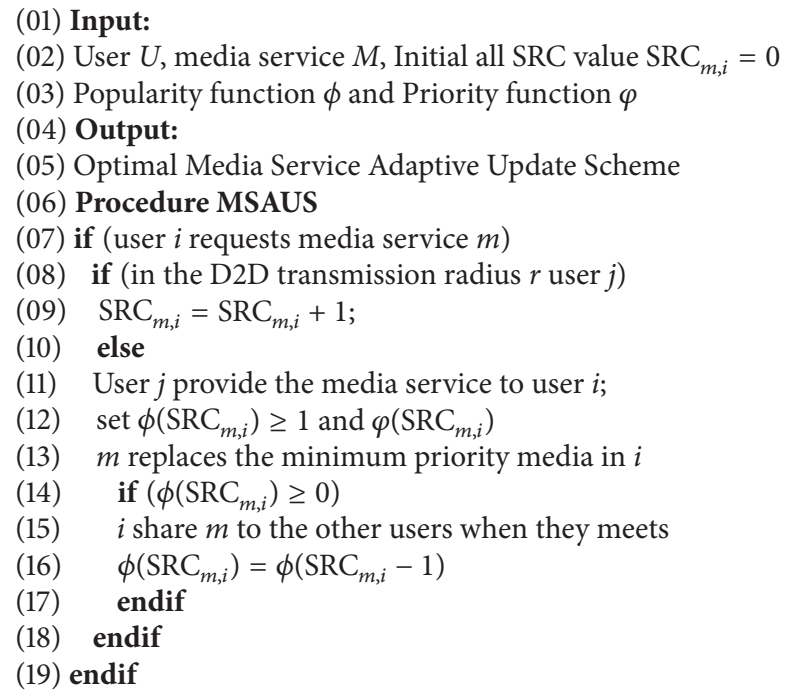

Algorithm 1: MSAUS scheme.

Then, we have

$$
\begin{aligned}
E(Q(\bar{\chi}))= & \left(1-e^{-\lambda \pi r^{2} m_{i} c}\right) Q_{d}(R, \Theta) \\
& +e^{-\lambda \pi r^{2} m_{i} c} Q_{c}(R, \Theta),
\end{aligned}
$$

where $E[\cdot]$ represents the expectation of random variable systems. According to the definition of the expected gain in the meeting slot $t$, we can see that each user QoE is mainly composed by D2D and cellular media service. For formula (10), only $m_{i}$ and $Q(R, \Theta)$ are variable for the certain networks. If we attempt to improve $E(Q(\bar{\chi}))$, we should adjust different media service distribution $m_{i}$ in the network according to different service QoE function $Q(R, \Theta)$.

Therefore, the value of the total user QoE MOS value can be summed for each media service $m$; that is,

$$
Q(\chi)=\sum_{m \in M} E(Q(\bar{\chi}))
$$

In the rest of this section, we attempt to design the priority and popularity function based on QoE function to design distributed media service scheme in Algorithm 1. This method might avoid the blindness of UE media service update and achieves a balance distributed service state in the network.

3.1. Expression of the Priority Function. For our proposed model, when user $i$ requests the demand service $m$, the user can pick up the media service from the vicinity users or the eNB for the higher QoE MOS value. It follows that if we desire to improve the expectations of QoE satisfaction of each user, $X_{i \in r}$ should contain more abundant media service $m$. On the other side, for the different media service distribution in the user's cache, it may regulate status according to $R$ and $\Lambda$. Thus, we define the heterogeneous media service priority function as

$$
\begin{aligned}
Q_{m}\left(\chi_{m}\right) \varphi\left(\chi_{m}\right) X_{i} & =Q_{n}\left(\chi_{n}\right) \varphi\left(\chi_{n}\right) X_{j} \\
& =\left(1-e^{-\lambda \pi r^{2} m_{i} c}\right) Q_{d}(R, \Theta) .
\end{aligned}
$$

In the process of media service priority estimate, no D2D transmission behavior occurs and the service update is a spontaneous behavior, which does not affect the user's satisfaction in the other service. Therefore, in the process of predicting service priority, we assume that the D2D communication interference is not considered, which means interference index $z=0$. However, the issue of service update caused by the decline in the quality of service within the same band will be discussed in the next section. Hence, according to (3), (4), and (5), $Q_{d}(R, \Theta)$ is represented as

$$
\begin{aligned}
R_{D}(d) & =B_{i} \log _{2}\left(1+\frac{p_{d} d_{u_{i}, u_{j}}^{-\alpha}\left|h_{d}\right|^{2}}{N_{0}}\right), \\
\Theta\left(R_{D}(d)\right) & =1-\left(e^{-\Lambda / R_{D}(d)}\left(\frac{\Lambda}{R_{D}(d)}\right)\right)^{Z-1}, \\
Q_{d}(R, \Theta) & =Q_{d}(d) .
\end{aligned}
$$

However, there are different QoE requirements for different media service in the Table 1, where $d$ is the user's Euclidean distance for $\mathrm{D} 2 \mathrm{D}$ pair under this scenario. The service in type T1, such as file downloading, is regardless of PER and MOS value decreases linearly with respect to $d$. T2 service, such as video streaming or conference D2D transmission is very sensitive to transmission distance $d$. Moreover, The marginal MOS value decrement of T3 service, such as lossless music service, becomes smaller as the distance increment. Examples of QoE functions for different type media service are shown in Figure 2. 


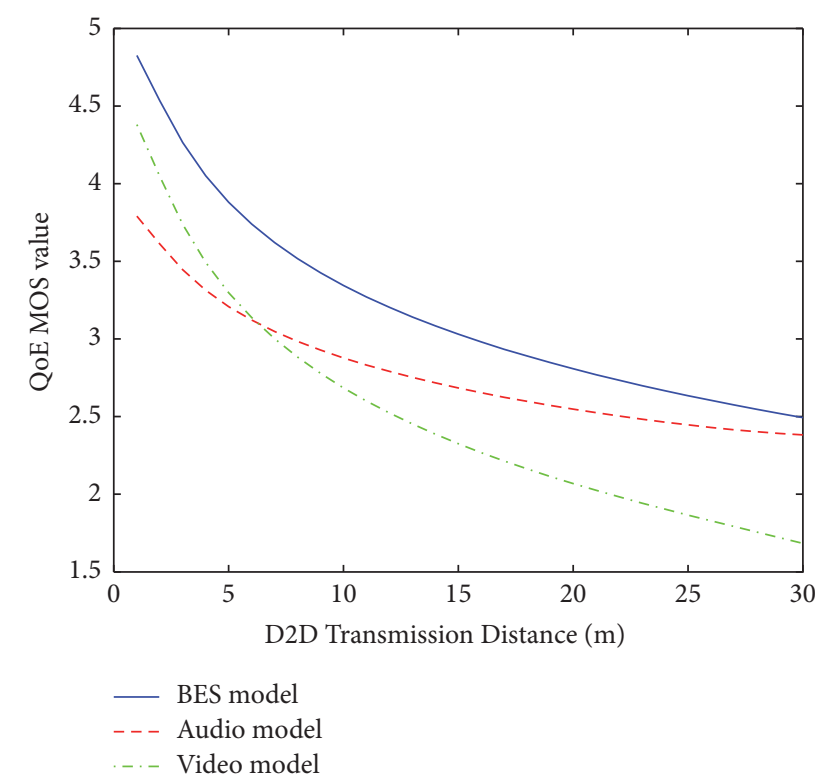

FIgUre 2: An example of QoE MOS value versus D2D distance for different type services.

Thereinto, the different types of media services obtain the same QoE MOS values $Q_{c}(R, \Theta)$ through the cellular link and can be considered as fixed constant. Meanwhile, it does not interact with the media service update in the cellular networks. Therefore, we mainly focus on user's satisfaction in D2D distributed scheme. Now, we obtain the probability distribution function of $m$ 's D2D radius; we use the decomposed function to model a dedicated user, that is,

$$
Q(\chi)=\sum_{m \in M} E\left(Q_{d}(\bar{d})\right)
$$

Thus, we define the heterogeneous media service priority function as

$$
Q_{m}\left(\chi_{m}\right) \varphi\left(\chi_{m}\right) X_{i}=Q_{n}\left(\chi_{n}\right) \varphi\left(\chi_{n}\right) X_{j}=E(Q(\tilde{\chi})) .
$$

This equation shows that $\partial Q / \partial X_{i}=E\left(Q_{d}(d)\right) e^{-\lambda \pi r^{2}} \varphi\left(\chi_{n}\right)$ where $\varphi$ is defined as

$$
\varphi(\tilde{\chi}) \propto \frac{e^{-\lambda \pi r^{2} \widetilde{X}_{i}}}{Q_{d}(d)} .
$$

Hence, we can set the priority function $\varphi(\tilde{\chi})$ as the order for the $u_{i}$ to rank the media service in his own cache and remove the media service with low priority; meanwhile, ensure the fairness for different media services.

3.2. Expression of the Popularity Function. We now describe the relationship between the priority function and the popularity function. Firstly, we give the expected value Service Receive Count (SRC) which is a measurement variable to $1 / X_{i}$ for service $m$ in UE $u$ when a UE meets others. There is roughly a probability $c \lambda m_{i}$ that the media service $m$ can be provided. Hence, we can set the UE's popularity function $\phi\left(|U| / X_{m}\right)$ as a first order of $X_{m}$. Meanwhile, for every user's cache, new media service always replace $m$ with probability $X_{i} / c|U|$. In addition, the media service propagation is inversely proportional to the proportion and number of media services $|M|$ for all the services. In a stable steady state, the appearance of new service is equal to deleted or replaced old service. Hence, we have

$$
\frac{c|U|}{X_{m}|M|} \phi\left(\frac{|U|}{X_{m}}\right)=\sum_{U} \frac{|U|}{X_{i}} \phi\left(\frac{|U|}{X_{i}}\right) .
$$

There are a lot of constants in the equation, and, therefore, we can write

$$
\frac{1}{X_{m}} \phi\left(\frac{1}{X_{m}}\right)=\frac{1}{X_{n}} \phi\left(\frac{1}{X_{n}}\right) \quad \forall m, n \in M .
$$

Alternatively, the scheme steady state should meet the equilibrium condition that we have

$$
\varphi(x)=\frac{M}{c x} \phi\left(\frac{|U|}{x}\right) \quad \forall x>0,
$$

where $\varphi$ is defined as shown above. It is easy to get the relationship between two functions. According to $\mathrm{SRC}_{m, i}$, the system achieves the maximum total user QoE value when the popularity function $\phi(m)$ and the priority function $\varphi(\tilde{\chi})$ satisfy [13]

$$
\phi(m)=\frac{c|U|}{|M|} \varphi\left(\frac{|U|}{m}\right) .
$$

On the basis of the relationship between the priority $\varphi(\tilde{\chi})$ and popularity function $\phi(m)$, the popularity function can be expressed as

$$
\phi\left(\mathrm{SRC}_{m, i}\right) \propto \frac{c|U| e^{-\lambda \mathrm{SRC}_{m, i}}}{\mathrm{SRC}_{m, i} \mathrm{Q}(d)} .
$$

Therein, $m_{i}|M|$ means the frequency of media service requested by users in the cellular networks. As the UE's service request is independent in the cell, we use $\mathrm{SRC}_{m, i}$ that can easily and explicitly calculate each user $i$ 's aspiration levels for media service $m$.

We indicate that the priority and popularity functions of each media service can be set adaptively to achieve the optimal and stable solution of the MSAUS scheme. Moreover, we summarize the expressions of the different priority and popularity functions for heterogeneous media service in the third and fourth rows of Table 1.

\section{Resource Management in the Different Media Service Delivery}

For different media services, the trade-off between the cellular resource and performance is also various. Therefore, the most prominent problem in $\mathrm{D} 2 \mathrm{D}$ communication is the interference between transmission [4]. How do we achieve a trade-off between the interference control issue and updating D2D interference? Here, in this part, we will go deeply into this problem. 


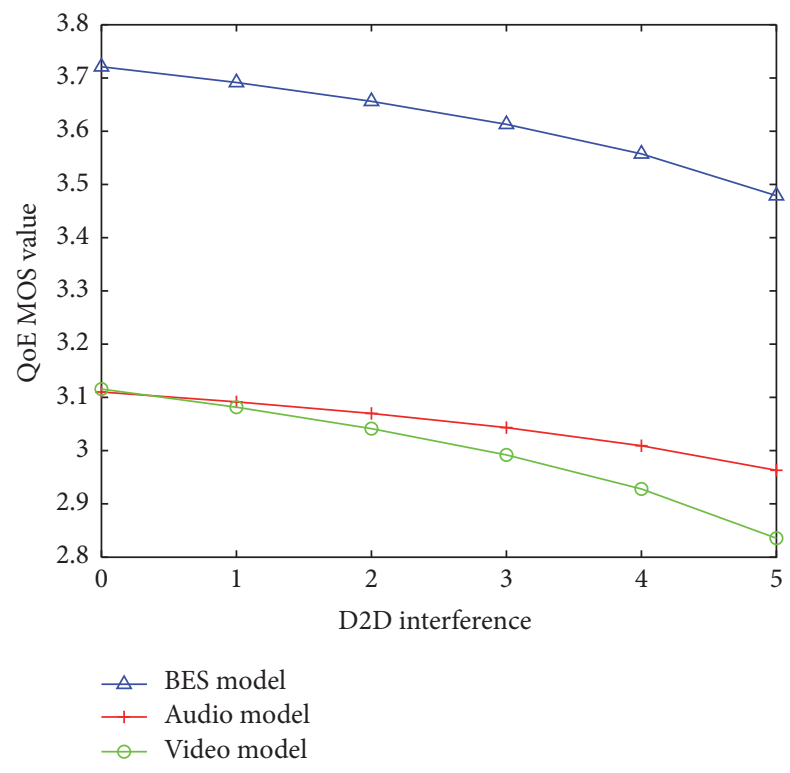

FIgURE 3: An example of QoE MOS value versus D2D interference $z$ for different type services.

We give an example of QoE functions versus D2D interference for different type media service in Figure 3. In Figure 3 , we can clearly perceive that the impact of D2D interference sources on the quality of multimedia services is very distinct. The increase of interference source has the greatest impact on the $\mathrm{T} 3$ service. Within the average increase of an interference source, the service quality will be decreased by $0.05 \mathrm{MOS}$ value. Therefore, for the different effects of $\mathrm{D} 2 \mathrm{D}$ interference on the service, the channel interference characteristics should be considered in the resources allocation $B_{i}$.

In order to ensure the transmission quality of the ongoing media service that the user requests, we propose a resource management scheme based on the media service popularity function. We call it Media Service Resource Allocation (MSRA) strategy. Note that we consider the trade-off between update cost and D2D interference to deploy limited bandwidth resource reasonably and efficiently. Each media service finds an appropriate resource allocation to render service or update it according to the popularity function $\phi$.

The core point of the MSRA strategy is to find the optimal bandwidth reuse in the cellular network. Each media service and user collaborate to mark the different bandwidth and avoid the tremendous interference to reduce the ongoing quality of service. And we also define $z_{i}$ to declare the $B_{i}$ bandwidth allocated condition to handle the usage of the different channel state. Based on the randomness of the user initiated request $m_{i}$ and uncertainty of heterogeneous services $T_{i}$, the general optimal algebraic optimization method is not suitable for this resource allocation problem in this situation. Therefore, in this paper we use the greedy algorithm to maximize the transmission performance $Q(\chi)$ of the cellular networks. The detailed operation of the MSRA is shown in Algorithm 2.

\section{Numerical Results}

In this section, we consider a cell network, where conventional UE is randomly distributed in the cell. Since the D2D users are usually within short distances, we adopt media service distribution model, where D2D users are uniformly distributed in a located circles and the simulation parameters are set according to [4]. The total users' QoE MOS value is used to evaluate the performance. Moreover, we compare our scheme with the LRU [5] (Least Recently Used) algorithm that is often used in the P2P networks and the typical network.

We first evaluate the performance of the MSAUS and MSRA scheme. Figure 4 compares the three schemes: the cellular network with MSAUS + MSRA, only with MSAUS, and LRU, when the subscriber number is 50, $z=5$ and three kinds of media service ratio are 1:1:1. From Figure 4, both users' QoE gain increases with the time slot. Thus, the MSAUS and MSRA scheme can achieve almost 9 performance gains compared to the only with MSAUS scheme. Then, MSAUS + MSRA and only MSAUS scheme can achieve almost 21 and 10 performance gains comparing with LRU algorithm, respectively. Due to the rapid interaction of users, in the balance, a large number of users that uses LRU algorithm have stored many of the same high popularity media service and deleted the relatively low prevalence media services. The scarcity cache resource utilization is extremely uneven in the networks, leading to further enhancement of the quality of media service difficultly. It is shown that although the LRU algorithm is suitable for the P2P network, the algorithm is not appreciate for the dynamic network.

For the imbalance media service situation, our proposed scheme has achieved a better QoE gain in the cellular networks, as in Figure 5. In particular, the maximum MOS value using the proposed scheme is 231.6 , whereas it is 217.3 only using MSAUS and 204.2 for the case of the LRU. This also shows that the proposed scheme, to the utmost extent, adapts to the environment which holds abundant higher QoE requested media services, like video or audio service. In view of the quality between different media services, the efficiency of the proposed algorithm is particularly prominent in the case of unbalanced media service distribution, and the problem of file popularity and scheduling of spectrum resources are of equal importance to improve the media service quality in the networks. In Figure 6, we repeat the results for different $z$. Note that $z$ only has certain influence on the increasing speed of user QoE, but for the stable state $z$ do not obtain more improvement to the total value. For the interference $z$ caused by the media service update, different types, and different priorities have a far-reaching impact on the performance of the service. It is very important to allocate a limited frequency band, but the number of bands $Z$ is only affected by the convergence speed of the service but has nothing to do with the convergence performance.

In Figure 7 we can clearly see that during the beginning of the system, the proposed scheme in this paper can quickly adjust the distribution of user services and improve the user satisfaction compared to the existing scheme. Moreover, in the case of different service distribution, when the popular 


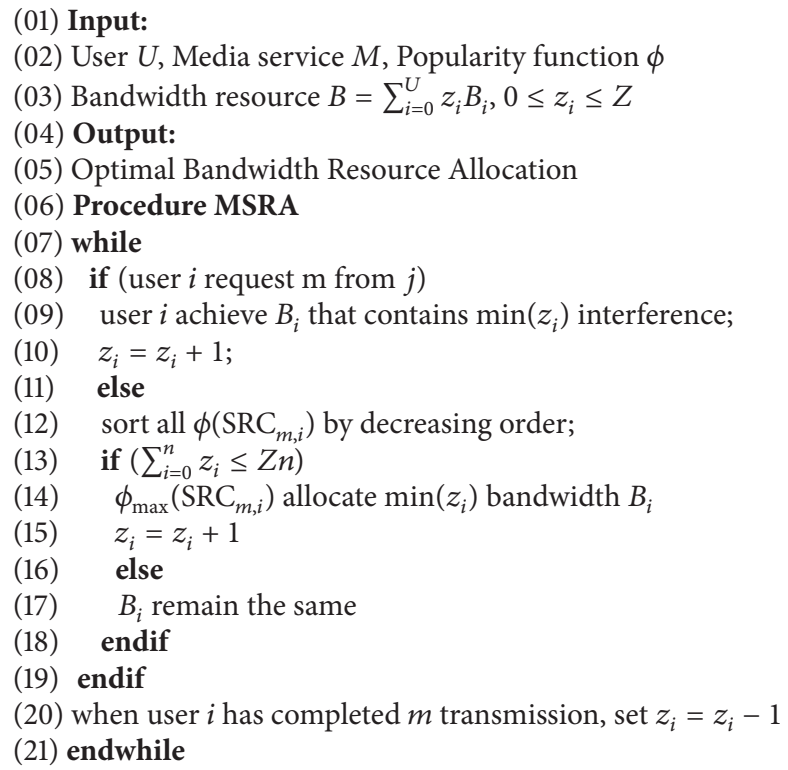

Algorithm 2: MSRA greedy scheme.

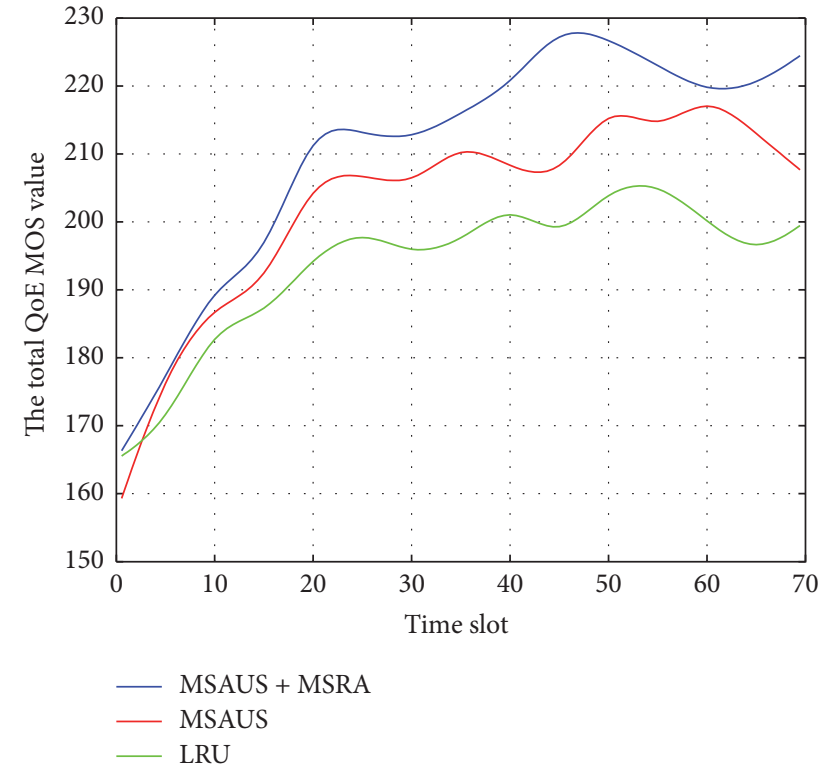

FIGURE 4: Performance comparison when BES: video: audio = $1: 1: 1$.

service is concentrated on few services, the system efficiency is more obvious. When $\gamma=0.8$, the average user's MOS value boosts $0.21 \mathrm{MOS}$ in the proposed scheme comparing to LRU scheme, which is almost equal to twice as much when $\gamma=0.4$. Figure 8 shows the media service D2D hit probability [18] for the case with two different schemes, respectively. As expected, the media service D2D hit probability increases in both schemes. Furthermore, we see the media service at the proposed scheme has much higher cache hit probability than caching at LRU scheme, as a result of service balance effect

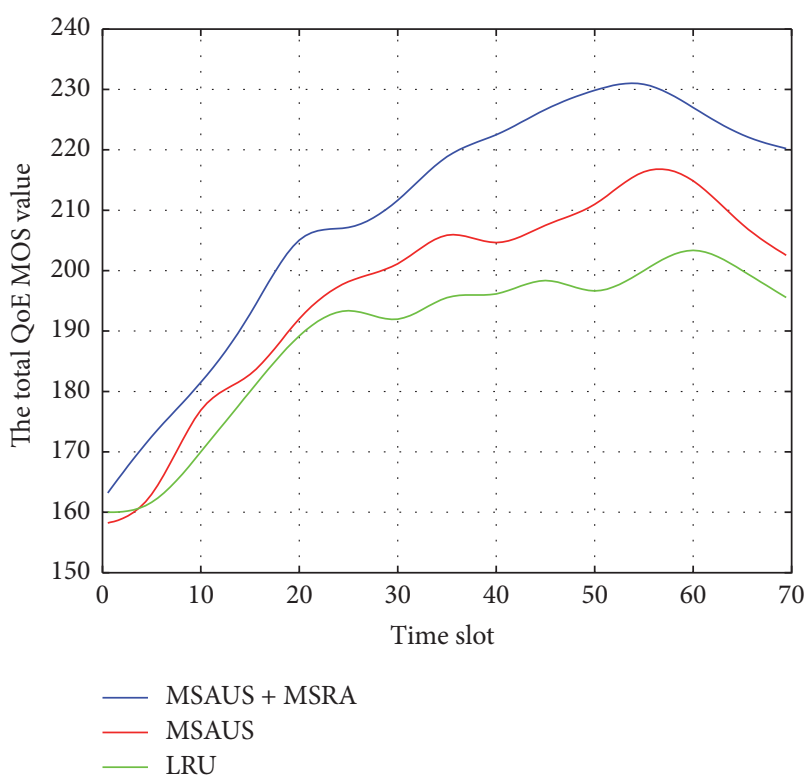

FIGURE 5: Performance comparison when BES: video: audio = $1: 2: 3$.

in priority function. The interaction between users makes the average MOS convergence faster; the rationality of media service storage leads to the higher D2D hit rate based on the algorithm we proposed in this paper.

\section{Conclusion}

In this paper, we proposed a distributed media service delivery and resource allocation scheme for D2D communication networks. Unlike the other conventional media service 


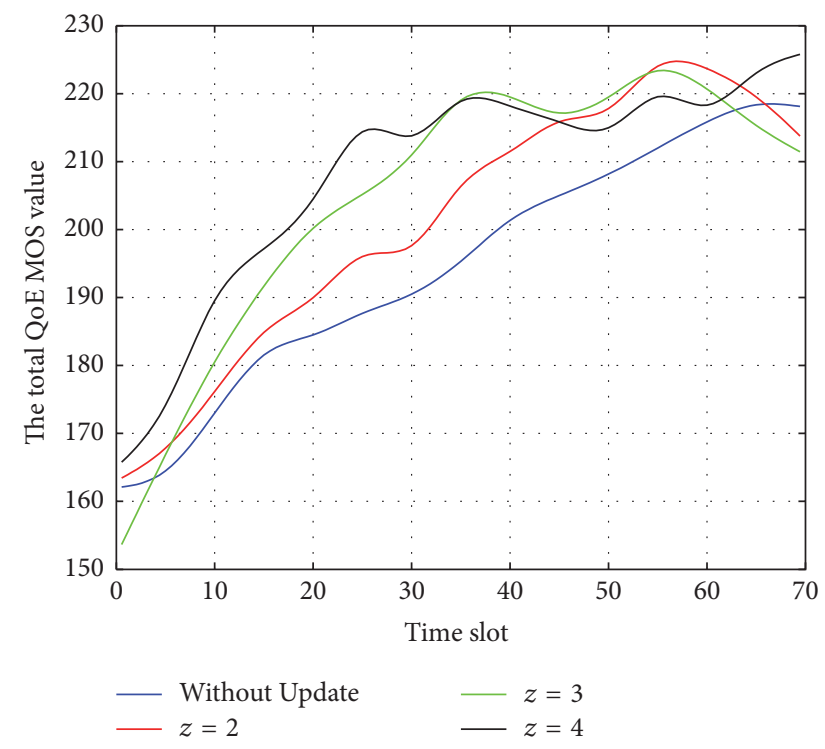

FIGURE 6: Performance comparison when $z$ increases and BES $:$ video $:$ audio $=1: 1: 1$.

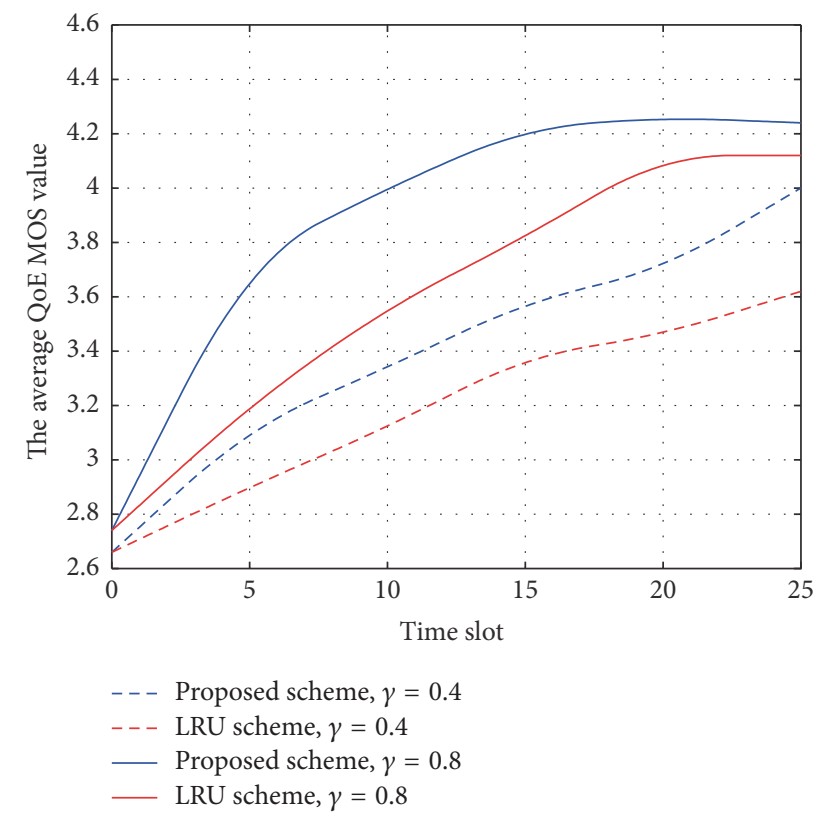

FIGURE 7: Performance comparison in the different media service popularity.

schemes or content delivery proposals, we do not focus on optimal quantity of service or throughput. Moreover, our work aims at achieving maximal total user's QoE value for dynamic cellular networks by jointly considering media service distribution and opportunistic transmission. Importantly, we combine the priority and popularity function, bandwidth allocation, and cache to achieve the goals of maximizing the total users' QoE value and solve the different content dissemination issues in D2D communication underlaying cellular networks. Our simulation results have

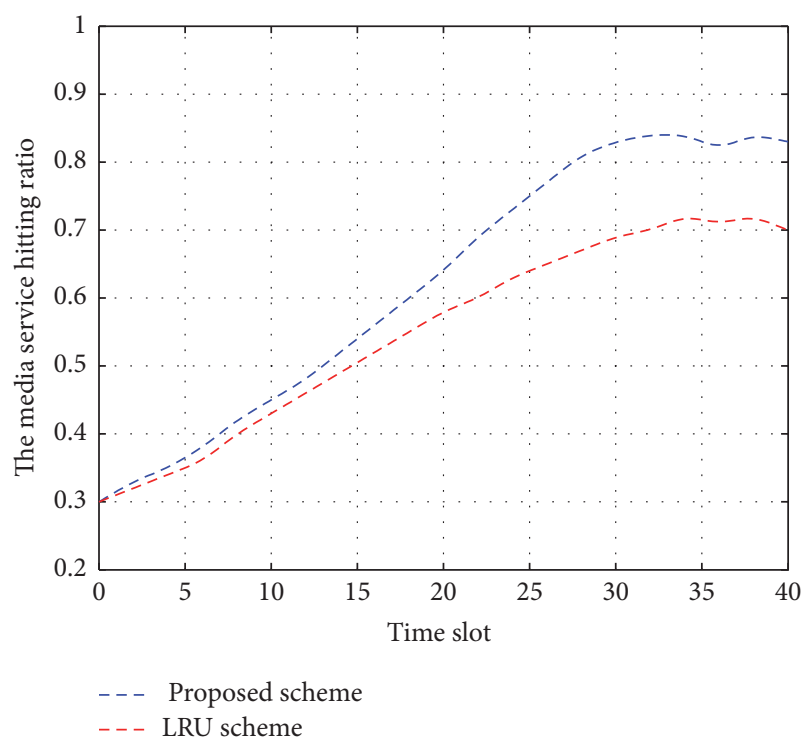

FIGURE 8: Probability of media service completion by D2D communication.

shown that the proposed scheme achieves even somewhat QoE promotion against the other schemes.

For practical heterogeneous media service in D2D communication underlaying cellular networks, additional work needs to be excavated. For instance, we attempt to develop a media content retrieval scheme to improve the user's QoE in the heterogeneous media service system and to study an optimal media service discovery method with blind UE meeting information. In our ongoing work, we plan to address more media service transmission characteristics and study their impact on the actual D2D networks.

\section{Notations}

$\left(u_{i}, U\right): \quad$ Mobile user, mobile user se

$(t, T): \quad$ Time slot, time

$\left(B_{i}, B\right): \quad$ Bandwidth resource

$z, Z: \quad$ Interference index, the max number of interference source

$m_{i}, M: \quad$ Media service, media service library

$\gamma: \quad$ A fixed parameter describes the skewness of media service popularity

$R_{c}, R_{D}$ : Data transmission rate between D2D links or cellular links

$p_{c}, p_{d}: \quad$ The transmission power from BS, the

$P_{C}, P_{D}: \quad$ The request distribution of D2D communication or cellular link

$\Lambda: \quad$ Packet exponent

$\Theta: \quad$ Packet error rate (PER)

$\lambda: \quad$ The density of Poisson distributed device random mobility

$X_{m, i}=(0,1):$ The indicator for possession of service $m$

$c: \quad$ Cache size

$Q(\chi): \quad$ MOS function for the media service 


\author{
$\phi: \quad$ Popularity function \\ $\varphi$ : $\quad$ Priority function \\ SRC: Service receive count \\ $d_{i, j}$ : Users Euclidean distance for D2D pair.
}

\section{Conflicts of Interest}

The authors declare that they have no conflicts of interest.

\section{Acknowledgments}

This work is partly supported by the National Natural Science Foundation of China (61571240), the Priority Academic Program Development of Jiangsu Higher Education Institutions, the Natural Science Foundation of Jiangsu Province (BK20161517), the Qing Lan Project, the Open Research Fund of Key Lab of Broadband Wireless Communication and Sensor Network Technology (NUPT), Ministry of Education (NYKL201509), the Open Research Fund of The State Key Laboratory of Integrated Services Networks, Xidian University (ISN17-04), The Major Projects of the Natural Science Foundation of the Jiangsu Higher Education Institutions (16KJA510004), the Natural Science Foundation of Jiangsu Province (Grant no. BK20161517), and the Postdoctoral Science Foundation of China (Grant no. 2017M611881).

\section{References}

[1] A. Zhang, J. Chen, R. Hu, and Y. Qian, "SeDS: secure data sharing strategy for D2D communication in LTE-advanced networks," IEEE Transactions on Vehicular Technology, vol. 65, no. 4, pp. 2659-2672, 2016.

[2] D. Wu, J. Wang, R. Q. Hu, Y. Cai, and L. Zhou, "Energy-efficient resource sharing for mobile device-to-device multimedia communications," IEEE Transactions on Vehicular Technology, vol. 63, no. 5, pp. 2093-2103, 2014.

[3] D. Wu, L. Zhou, Y. Cai, R. Hu, and Y. Qian, "The role of mobility for D2D communications in LTE-advanced networks: energy vs. bandwidth efficiency," IEEE Wireless Communications, vol. 21, no. 2, pp. 66-71, 2014.

[4] D. Feng, L. Lu, Y.-W. Yi, G. Y. Li, G. Feng, and S. Li, "Device-todevice communications underlaying cellular networks," IEEE Transactions on Communications, vol. 61, no. 8, pp. 3541-3551, 2013.

[5] L. Zhou, R. Q. Hu, Y. Qian, and H.-H. Chen, "Energy-spectrum efficiency tradeoff for video streaming over mobile ad hoc networks," IEEE Journal on Selected Areas in Communications, vol. 31, no. 5, pp. 981-991, 2013.

[6] C. Xu, F. Zhao, J. Guan, H. Zhang, and G.-M. Muntean, "QoEdriven user-centric vod services in urban multihomed P2Pbased vehicular networks," IEEE Transactions on Vehicular Technology, vol. 62, no. 5, pp. 2273-2289, 2013.

[7] C. Xu, S. Jia, L. Zhong, H. Zhang, and G.-M. Muntean, "Ant-inspired mini-community-based solution for video-ondemand services in wireless mobile networks," IEEE Transactions on Broadcasting, vol. 60, no. 2, pp. 322-335, 2014.

[8] N. Golrezaei, P. Mansourifard, A. F. Molisch, and A. G. Dimakis, "Base-station assisted device-to-device communications for high-throughput wireless video networks," IEEE Transactions on Wireless Communications, vol. 13, no. 7, pp. 3665-3676, 2014.

[9] N. Golrezaei, A. G. Dimakis, and A. F. Molisch, "Scaling behavior for device-to-device communications with distributed caching," IEEE Transactions on Information Theory, vol. 60, no. 7, pp. 4286-4298, 2014.

[10] N. Golrezaei, A. Molisch, A. G. Dimakis, and G. Caire, "Femtocaching and device-to-device collaboration: a new architecture for wireless video distribution," IEEE Communications Magazine, vol. 51, no. 4, pp. 142-149, 2013.

[11] Y. Li, Z. Wang, D. Jin, and S. Chen, "Optimal mobile content downloading in device-to-device communication underlaying cellular networks," IEEE Transactions on Wireless Communications, vol. 13, no. 7, pp. 3596-3608, 2014.

[12] H. Zhu, Y. Cao, W. Wang, B. Liu, and T. Jiang, "QoE-aware resource allocation for adaptive device-to-device video streaming," IEEE Network, vol. 29, no. 6, pp. 6-12, 2015.

[13] L. Zhou, Y. Zhang, K. Song, W. Jing, and A. V. Vasilakos, "Distributed media services in P2P-based vehicular networks," IEEE Transactions on Vehicular Technology, vol. 60, no. 2, pp. 692-703, 2011.

[14] L. Zhou, Z. Yang, Y. Wen, H. Wang, and M. Guizani, "Resource allocation with incomplete information for QoE-driven multimedia communications," IEEE Transactions on Wireless Communications, vol. 12, no. 8, pp. 3733-3745, 2013.

[15] L. Zhou, "Mobile device-to-device video distribution: theory and application," ACM Transactions on Multimedia Computing, Communications and Applications, vol. 12, no. 3, article 38, 2016.

[16] J. Ning, S. Singh, K. Pelechrinis, B. Liu, S. V. Krishnamurthy, and R. Govindan, "Forensic analysis of packet losses in wireless networks," IEEE/ACM Transactions on Networking, vol. 24, no. 4, pp. 1975-1988, 2016.

[17] J. Rao, H. Feng, C. Yang, Z. Chen, and B. Xia, "Optimal caching placement for D2D assisted wireless caching networks," in Proceedings of the IEEE International Conference on Communications (ICC '16), pp. 1-6, Kuala Lumpur, Malaysia, May 2016.

[18] I. Pappalardo, G. Quer, B. D. Rao, and M. Zorzi, "Caching strategies in heterogeneous networks with D2D, small BS and macro BS communications," in Proceedings of the IEEE International Conference on Communications (ICC '16), pp. 1-6, Kuala Lumpur, Malaysia, May 2016. 


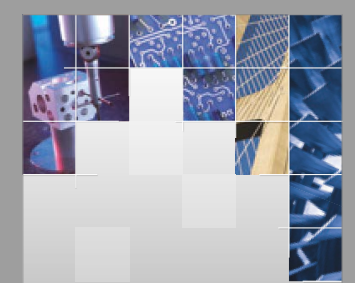

\section{Enfincering}
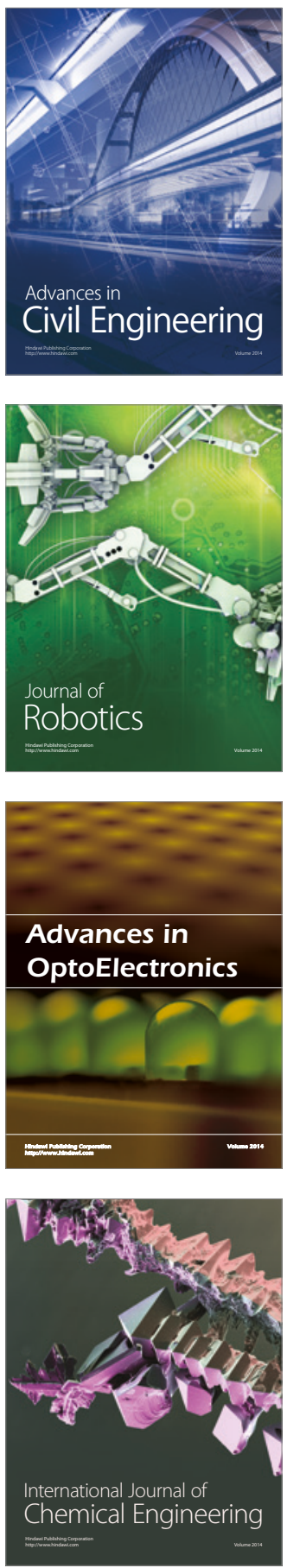

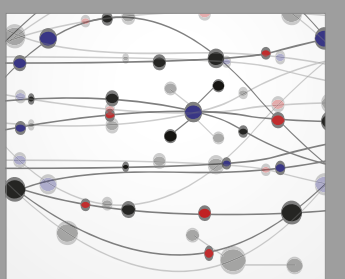

The Scientific World Journal

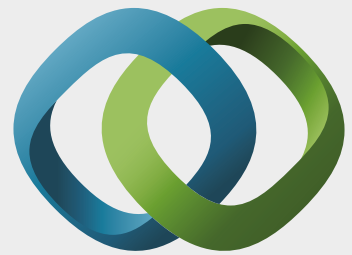

\section{Hindawi}

Submit your manuscripts at

https://www.hindawi.com
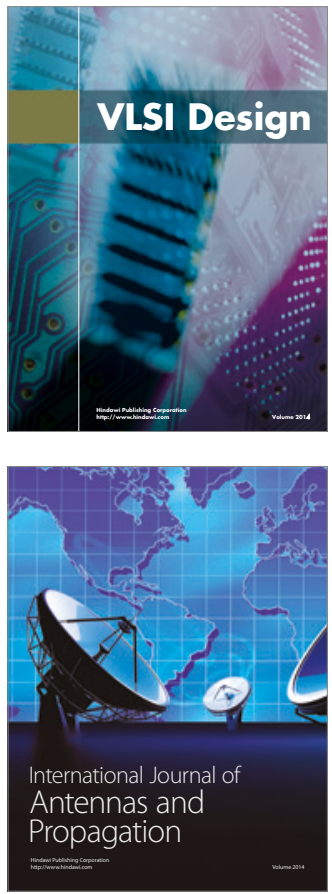

\section{Rotating}

Machinery
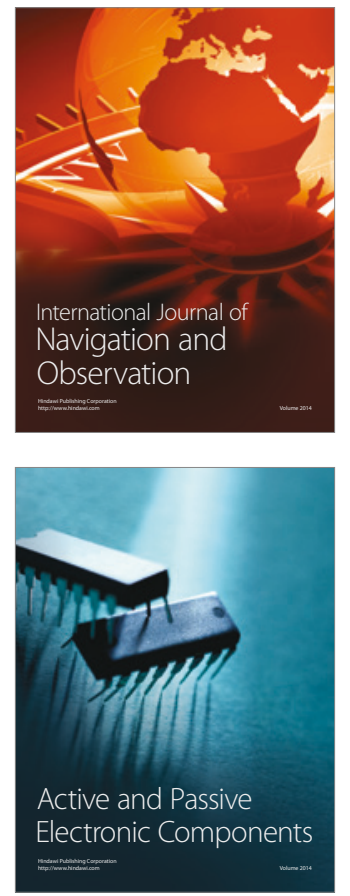
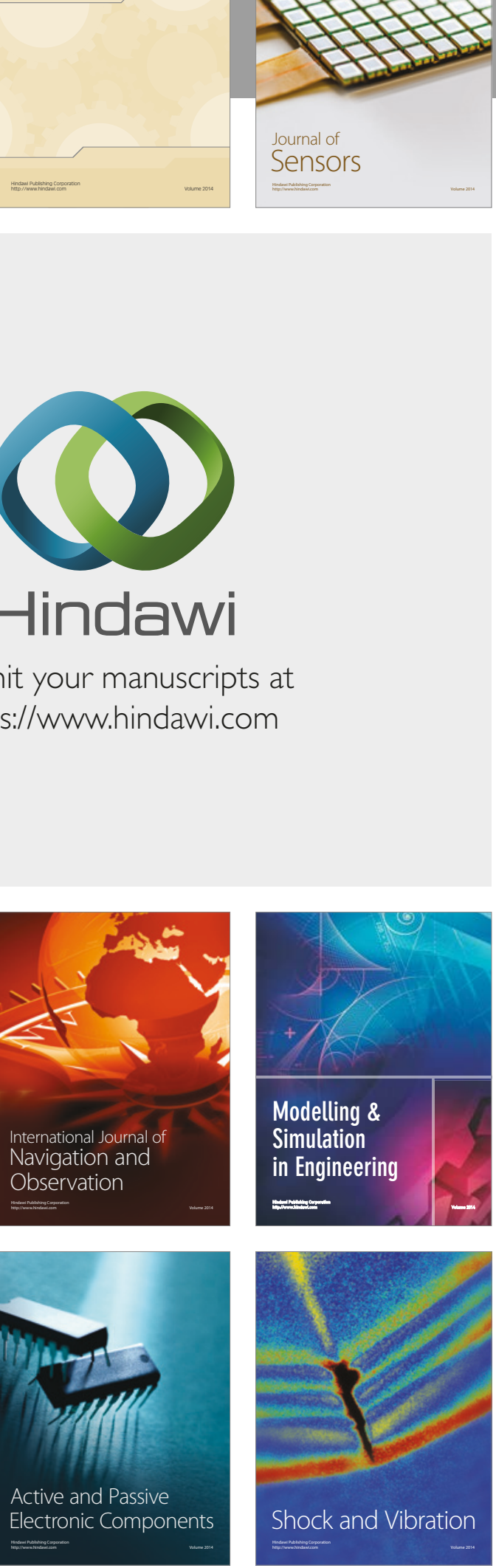
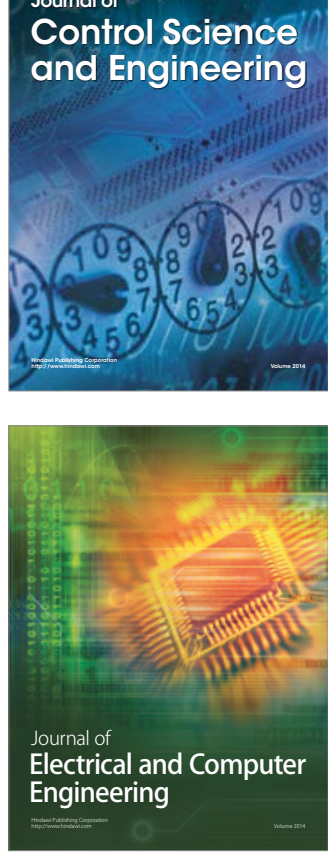

Distributed

Journal of

Control Science

and Engineering
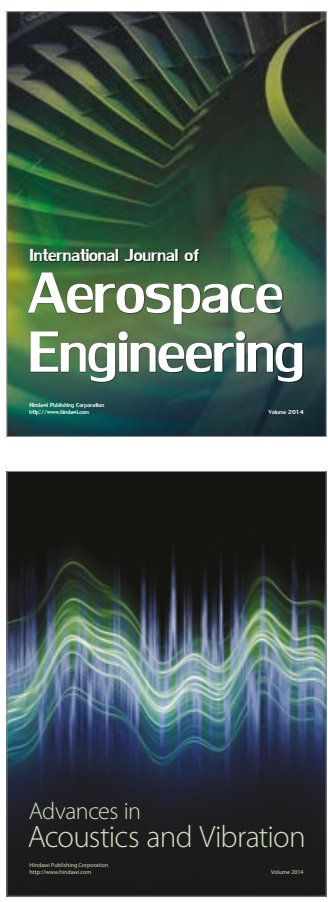

Sensor Networks 